\title{
X-ray constrained Spin-Coupled wavefunction: a new tool to extract chemical information from X-ray diffraction data
}

\author{
Alessandro Genoni, ${ }^{[a]}$ Davide Franchini, ${ }^{[b]}$ Stefano Pieraccini,${ }^{[c]}$ and Maurizio Sironi ${ }^{[c]}$
}

\begin{abstract}
The X-ray constrained wavefunction (XCW) approach is a reliable and widely used method of Quantum Crystallography that allows the determination of wavefunctions compatible with X-ray diffraction data. So far, all the existing XCW techniques have been developed in the framework of the Molecular Orbital theory and consequently, provide only pictures of the "experimental" electronic structures that are far from the traditional chemical perception. Here we propose a new strategy that, by combining the XCW philosophy with the Spin-Coupled method of the Valence Bond theory, enables to directly extract traditional chemical information (e.g., weights of resonance structures) from X-ray diffraction measurements. Preliminary results have shown that the new technique is really able to efficiently capture the effects of the crystal environment on the electronic structure and can be considered as a new useful tool to perform chemically sound analyses of the X-ray diffraction data.
\end{abstract}

Besides the well-known multipolar model, ${ }^{[1]}$ one of the new pillarmethods of the emerging field of Quantum Crystallography ${ }^{[2]}$ is Jayatilaka's X-ray constrained wavefunction (XCW) approach, ${ }^{[3]}$ which is the most widely used strategy among the techniques that aim at extracting wavefunctions or density matrices from experimental X-ray diffraction or scattering data. ${ }^{[4]}$ It consists in determining wavefunctions that not only minimize the energy of the investigated systems, but that also maximize the agreement between calculated and experimental structure factors amplitudes. So far, the approach has been mainly proposed within the Molecular Orbital theory, especially in the framework of the Hartree-Fock formalism, ${ }^{[3]}$ but also including relativistic corrections. $^{[5]}$ Therefore, in its original form, the Jayatilaka strategy provides completely delocalized pictures of electronic structures that can be rationalized in terms of traditional chemical concepts (e.g., local bonds, hybridization, Lewis structures, resonance structures, etc.) by only applying a posteriori Quantum Chemical Topology methods ${ }^{[6,7]}$ or other bonding-analysis techniques, such as the Natural Bond Orbitals strategy and the Natural Resonance Theory. ${ }^{[7]}$ The only attempts

[a] Dr. A. Genoni

Université de Lorraine, CNRS, Laboratoire LPCT

1 Boulevard Arago, F-57078, Metz (France)

E-mail: Alessandro.Genoni@univ-lorraine.fr

[b] D. Franchini

Dipartimento di Chimica

Università degli Studi di Milano

Via Golgi 19, 20133, Milano (Italy)

[c] Dr. S. Pieraccini, Prof. Dr. M. Siron

Dipartimento di Chimica

Università degli Studi di Milano

Via Golgi 19, 20133, Milano (Italy)

and

Istituto di Scienze e Tecnologie Molecolari (ISTM), CNR

Via Golgi 19, 20133, Milano (Italy)

and

Consorzio Interuniversitario Nazionale per la Scienza e Tecnologia

dei Materiali (INSTM), UdR Milano

Via Golgi 19, 20133, Milano (Italy)

Supporting information for this article is available on the Internet under http://dx.doi.org/10.1002/chem.2018xxxxx. to recover the traditional chemical perception in the context of the XCW approach without resorting to a posteriori methods are the more recent $\mathrm{XC}-\mathrm{ELMO}^{[8]}$ and $\mathrm{XC}-\mathrm{ELMO} \mathrm{VB}^{[9]}$ techniques. Nevertheless, in the former, Extremely Localized Molecular

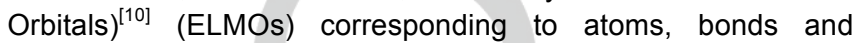
functional groups are directly extracted from X-ray diffraction data, but a localization scheme is actually imposed a priori on the electronic structure before starting the calculations. In the latter, only the weights of resonance structures are determined from the experimental data, while the Slater determinants used for the expansion of a pseudo Valence Bond wavefunction are obtained by means of preliminary ELMO calculations and are strictly kept frozen during the minimization-fitting process.

Here we present a novel XCW method that allows the extraction of usual chemical information from X-ray diffraction data without performing a posteriori analyses or introducing a priori information. To accomplish this task we have coupled the XCW philosophy to the Spin-Coupled (SC) approach, ${ }^{[11]}$ a Valence Bond ${ }^{[12]}$ technique that provides a fully correlated description of the electronic structure, but still interpretable in terms of oneparticle functions and close to the traditional chemical perception. In fact, from the shapes of the SC orbitals it is generally possible to draw conclusions on the spatial arrangements of the electronic clouds and the hybridization of atoms. Furthermore, from the weights associated with the different spin-coupling modes, one can get insights into the relative importance of the resonance structures for the system under exam.

In the new X-ray constrained Spin-Coupled (XCSC) technique, the wavefunction ansatz assumes the analytical form of a traditional SC wavefunction for a system of $N$ electrons in the spin-state $(S, M)$ :

$$
\Psi_{0}=\sum_{k=1}^{f_{S}^{N_{v}}} c_{S, k} \psi_{S, M ; k}^{N}=\sum_{k=1}^{f_{S}^{N_{v}}} c_{S, k} \mathcal{A}\left(\phi_{1}^{c} \bar{\phi}_{1}^{c} \ldots \phi_{i}^{c} \bar{\phi}_{i}^{c} \ldots \phi_{N_{1}}^{c} \bar{\phi}_{N_{1}}^{c} \boldsymbol{\Phi}_{\mathrm{v}} \Theta_{S, M ; k}^{N_{v}}\right)
$$

where $S$ and $M$ are the quantum numbers associated with the spin operators $\hat{S}^{2}$ and $\hat{S}_{z}$, respectively, $\mathcal{A}$ is the usual antisymmetrizer, $f_{S}^{N_{v}}$ is the number of linearly independent spineigenfunctions (and, equivalently, of the linearly independent Spin-Coupled structures) for the system under exam given by

$$
f_{S}^{N_{v}}=\frac{(2 S+1) N !}{\left(\frac{1}{2} N+S+1\right) !\left(\frac{1}{2} N-S\right) !}
$$

$\psi_{S, M ; k}^{N}$ is the $k$-th Spin-Coupled structure, $\Theta_{S, M ; k}^{N}$ is the $k$-th spineigenfunction and $\left\{c_{S, k}\right\}$ are the spin-coupling coefficients that provide the weights of the Spin-Coupled structures $\left\{\psi_{S, M ; k}^{N}\right\}$ in wavefunction $\Psi_{0}$. As in any SC calculation, ${ }^{[11]}$ in order to reduce the large computational cost, the $N$ electrons of the investigated system are subdivided into two groups: a subset of $2 N_{1}$ core (or inactive) electrons and a subset of $N_{v}$ valence (or active) electrons. The former are described by frozen doubly occupied Molecular Orbitals previously obtained through a Hartree-Fock computation, while the latter are those really treated at SpinCoupled level. For this reason, in equation (1), $\phi_{i}^{c}$ is a frozen "core spin-orbital" with spatial part $\phi_{i}^{c}$ and spin part $\alpha, \bar{\phi}_{i}^{c}$ is a 
frozen "core spin-orbital" with spatial part $\phi_{i}^{c}$ and spin part $\beta$, and $\boldsymbol{\Phi}_{\mathrm{V}}$ is the product of the $N_{v}$ "active Spin-Coupled orbitals", namely:

$$
\boldsymbol{\Phi}_{\mathrm{v}}\left(\boldsymbol{r}_{1}, \boldsymbol{r}_{2}, \ldots \boldsymbol{r}_{N_{v}}\right)=\phi_{1}\left(\boldsymbol{r}_{1}\right) \phi_{2}\left(\boldsymbol{r}_{2}\right) \ldots \phi_{N_{v}}\left(\boldsymbol{r}_{N_{v}}\right)
$$

Due to the non-orthogonality of the Spin-Coupled structures in equations (1), the real weights of structures $\left\{\psi_{S, M ; k}^{N}\right\}$ are not given by the spin coupling coefficients $\left\{c_{S, k}\right\}$, but by the corresponding Chirgwin-Coulson coefficients, ${ }^{[13]}$ which are defined like this:

$$
w_{S, k}=\left|c_{S, k}\right|^{2}+\sum_{j \neq k} c_{S, k} c_{S, j} S_{k j}
$$

where $S_{k j}$ is the overlap integral between Spin-Coupled structures $\psi_{S, M ; k}^{N}$ and $\psi_{S, M ; j}^{N}$.

Using equation (1) as ansatz, the goal of the new XCW technique is to find the active Spin-Coupled orbitals $\left\{\phi_{i}\right\}$ and the spin-coupling coefficients $\left\{c_{S, k}\right\}$ that minimize the functional:

$$
J\left[\left\{\phi_{i}\right\},\left\{c_{S, k}\right\}\right]=W\left[\left\{\phi_{i}\right\},\left\{c_{S, k}\right\}\right]+\lambda\left(\chi^{2}\left[\left\{\phi_{i}\right\},\left\{c_{S, k}\right\}\right]-\Delta\right)
$$

The first term $W$ is the energy part of the functional, namely

$$
W\left[\left\{\phi_{i}\right\},\left\{c_{S, k}\right\}\right]=\frac{\left\langle\Psi_{0}\left|\widehat{H}_{0}\right| \Psi_{0}\right\rangle}{\left\langle\Psi_{0} \mid \Psi_{0}\right\rangle}
$$

with $\widehat{H}_{0}$ as the non-relativistic Hamiltonian operator for the reference crystal-unit (see Supporting Information for more details), while the second term takes into account the constraint of the experimental data. In particular, $\lambda$ is an external multiplier that is manually adjusted during the calculations and that gives the strength of the experimental constraints, $\Delta$ is the desired agreement between theoretical and observed values (usually set equal to 1) and $\chi^{2}$ represents a measure of the statistical agreement between the calculated and the theoretical structure factors amplitudes:

$$
\chi^{2}=\frac{1}{N_{r}-N_{p}} \sum_{h} \frac{\left(\eta\left|F_{h}^{\text {calc }}\right|-\left|F_{h}^{\text {exp }}\right|\right)^{2}}{\sigma_{h}^{2}}
$$

with $N_{r}$ as the number of reflections used as constrains, $N_{p}$ as the number of adjustable parameters, $\boldsymbol{h}$ as the triad of Miller indexes labeling the reflection, $\sigma_{h}$ as the experimental uncertainty associated with the generic experimental structure factor amplitude $\left|F_{h}^{\text {exp }}\right|$ and $\eta$ as an overall scale-factor, which is determined by minimizing the $\chi^{2}$ value and which multiplies each computed structure factor amplitude $\left|F_{h}^{\text {calc }}\right|$.

The XCSC technique has been tested exploiting the highresolution X-ray diffraction data collected for benzene at $100 \mathrm{~K}$ by Bürgi and coworkers. ${ }^{[14]}$ Using the crystallographic geometry we have carried out both traditional and X-ray constrained SpinCoupled calculations with the DZP-Dunning basis-set to expand the orbitals. For both type of Spin-Coupled computations, the 6 $\pi$ electrons of the aromatic ring have been considered as active electrons and have thus been described through SC orbitals, while both the core electrons of the carbon atoms and the electrons associated with the $\mathrm{C}-\mathrm{H}$ and $\mathrm{C}-\mathrm{C} \sigma$ bonds of the molecule have been described with frozen doubly occupied molecular orbitals initially obtained from Restricted Hartree-Fock calculations. According to equation (2), the 6 active electrons of the system in the singlet state have been spin-coupled in 5 different ways, which correspond to the 5 possible resonance structures of benzene shown in Figure 1. The first two correspond to the traditional Kekulé resonance structures (Figures $1 \mathrm{~A}$ and $1 \mathrm{~B}$ ), while the other three are those of Dewar (Figures 1C-1E). Finally, only for the XCSC calculations, we have exploited the unit-cell data, the Anisotropic Displacement Parameters (ADPs) and all the structure factors amplitudes associated with the crystallographic structure of benzene (particularly, 2412 structure factor amplitudes used as constraints). The X-ray constrained calculations have been carried out by progressively varying the external multiplier $\lambda$ from 0 (unconstrained SC calculation) with steps of 0.05 . As termination criteria for the XCSC calculations we have adopted those already proposed in the framework of the XC-ELMO method $^{[8 b-8 \mathrm{~d}]}$ (see Supporting Information).

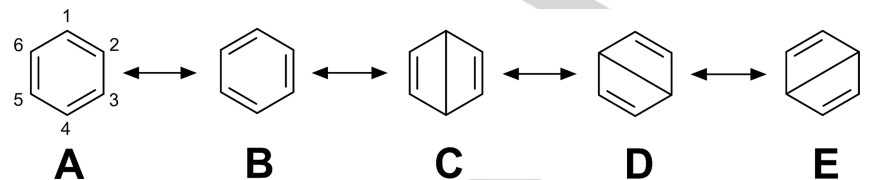

Figure 1. Spin-Coupled resonance structures of benzene taken into account in the unconstrained and X-ray constrained Spin-Coupled calculations (A-B: Kekulé resonance structures; C-E: Dewar resonance structures). The adopted labels for the carbon atoms of the aromatic ring are also shown in structure A.

After performing the XCSC calculations, we have initially considered the trend of $\chi^{2}$ in function of the external multiplier $\lambda$ (see Figure 2). As in any X-ray constrained wavefunction computation, the statistical agreement decreases monotonically as $\lambda$ increases and the first steps are the ones characterized by the sharpest decreases in the $\chi^{2}$ value. Exploiting the adopted termination criteria, the XCSC calculations have been halted at $\lambda$ $=0.40$, as indicated by the vertical red dotted line depicted in Figure 2.

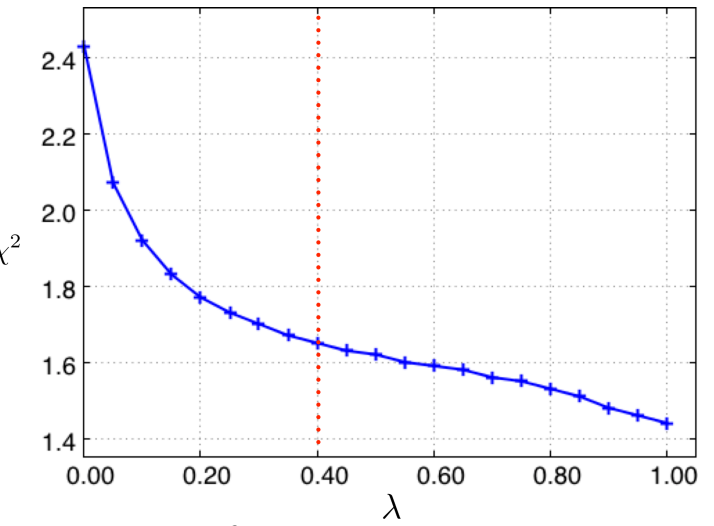

Figure 2. Variation of the $\chi^{2}$ statistical agreement in function of the external multiplier $\lambda$ for the XCSC calculations.

Table 1. $\chi^{2}$ statistical agreement and electronic energy values for the analyzed unconstrained and X-ray constrained Spin-Coupled wavefunctions

\begin{tabular}{lcc}
\hline Method & $\chi^{2}$ & Energy $\left(E_{h}\right)$ \\
\hline Spin-Coupled & 2.43 & -230.808252 \\
$\operatorname{XCSC}(\lambda=0.20)$ & 1.77 & -230.778317 \\
$\operatorname{XCSC}(\lambda=0.40)$ & 1.65 & -230.752291 \\
\hline
\end{tabular}

For the sake of completeness, the $\chi^{2}$ values resulting from the unconstrained and X-ray constrained Spin-Coupled calculations are reported in Table 1 together with the corresponding electronic energies. As already observed for other XCW strategies, the energy associated with the experimentally constrained wavefunction is higher than the corresponding unconstrained energy. This is due to the fact that, when we determine an X-ray constrained Spin-Coupled wavefunction, additional constraints are introduced without taking into account new variational parameters. Therefore, the obtained SpinCoupled orbitals and spin-coupling coefficients correspond to a minimum point on the hyper-surface of functional (5) and not to a minimum point for the energy of the system. 

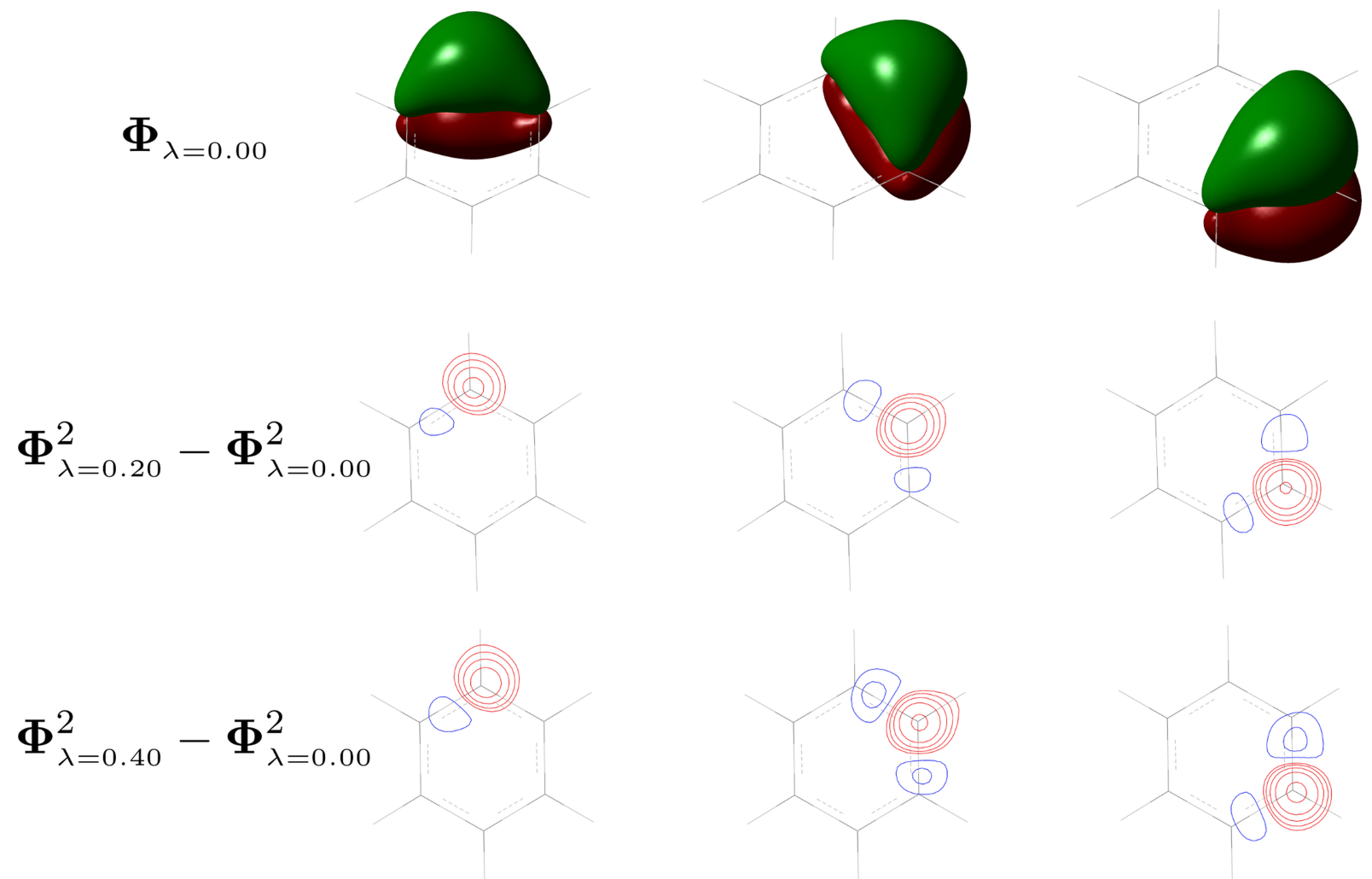

Figure 3. First row: three-dimensional plots of the symmetry unique unconstrained Spin-Coupled orbitals $\left(0.06\right.$ e/bohr ${ }^{3}$ isovalues are plotted). Second row: twodimensional plots of the differences between the square moduli of the X-ray constrained $(\lambda=0.20)$ and unconstrained Spin-Coupled orbitals. Third row: twodimensional plots of the differences between the square moduli of the X-ray constrained $(\lambda=0.40)$ and unconstrained Spin-Coupled orbitals. For each twodimensional plot, the contours are drawn at $\pm 1 \cdot 10^{-3}$ e/bohr ${ }^{3}$ and at $\pm 2,4,8 \cdot 10^{n}$ e/bohr ${ }^{3}$ (with $n$ as an integer ranging from -3 to 0 ) in a plane parallel to and $0.5 \AA$ above the one defined by the carbon atom on which the orbital is mainly localized and the other two carbon atoms bonded to it. Red and blue contours indicate positive and negative values, respectively.

We have afterwards studied more in detail the effects of the experimental constraints on the Spin-Coupled wavefunction by comparing the results of the unconstrained and $\mathrm{X}$-ray constrained Spin-Coupled calculations. In order to better show how the effects of the X-ray diffraction data are progressively taken into account in the computations, we have considered not only the final XCSC wavefunction at $\lambda=0.40$, but also the intermediate one obtained for $\lambda=0.20$. We initially focused on the Spin-Coupled orbitals and, in Figure 3, other than depicting the unconstrained (symmetry unique) ones, we have also shown their differences with those resulting from the X-ray constrained calculations. For all the orbitals, the main effect of the experimental X-ray diffraction data is a shift of the electronic clouds from the bonding regions to the carbon atoms. In other words, the XCSC orbitals are more localized on the carbon atoms than the corresponding unconstrained ones. The effect becomes more significant as $\lambda$ increases.

At a second stage we have also focused on the spin-coupling coefficients and, more particularly, on the corresponding Chirgwin-Coulson weights that directly measure the relative importance of the different resonance structures (see Table 2). In gas phase (namely, in the unconstrained Spin-Coupled wavefunctions) the resonance structures that have a predominant weight are the Kekule ones, each of them contributing for about $40 \%$, which is fully consistent with the results of SC calculations performed on benzene molecular geometries optimized in gas-phase. ${ }^{[11 \mathrm{c}, 11 \mathrm{~d}]}$ Nevertheless, as we introduce the experimental constraints in the computations, structures A and B still remain the most significant, but they gradually lose their importance in favour of Dewar resonance structure $\mathrm{E}$, whose weight passes from about $6 \%$ in gas phase to $9 \%$ in the intermediate XCSC wavefunction $(\lambda=0.20)$ and to about $11 \%$ in the final XCSC wavefunction $(\lambda=0.40)$.

Table 2. Chirgwin-Coulson weights (in \%) of the benzene Spin-Coupled resonance structures, as obtained from unconstrained $(\lambda=0.0)$ and X-ray constrained Spin-Coupled calculations.

\begin{tabular}{lccc}
\hline & \multicolumn{3}{c}{ Chirgwin-Coulson weights } \\
\cline { 2 - 4 } Resonance Structure & $\lambda=0.0$ & $\lambda=0.20$ & $\lambda=0.40$ \\
\hline A & 40.45 & 39.13 & 38.22 \\
B & 40.45 & 39.17 & 38.26 \\
C & 6.34 & 5.90 & 5.63 \\
D & 6.23 & 6.79 & 6.76 \\
E & 6.53 & 9.01 & 11.12 \\
\hline
\end{tabular}

To further study the effects of the wavefunction fitting, we have also compared the unconstrained Spin-Coupled electron densities with the XCSC ones obtained for $\lambda=0.20$ and $\lambda=$ 0.40 . In Figure 4, we have plotted the differences between the $\mathrm{X}$-ray constrained electron distributions and the reference unconstrained Spin-Coupled electron distributions both on a plane above the aromatic ring (first row in Figure 4) and on a plane below the aromatic ring (second row in Figure 4). The reason why we plotted the differences both above and below the benzene aromatic ring is to show that the difference-maps fully respect the symmetry of the inversion center of the molecule. In fact, the benzene crystallographic geometry used for our calculations is not exactly planar and the plots only on one side of the ring result in non-centrosymmetric pictures. However, the differences shown in Figure 4 indicate that the introduction of the 
constraint of the X-ray diffraction data entails a redistribution of the molecular electron density, mainly from the C-C bonds to the carbon atoms, thus confirming what we have already observed by analyzing the differences between the unconstrained and $X$ ray constrained SC orbitals. Also in this case, the entity of the charge redistribution increases as the experimental constraints become more and more important in the functional to minimize.
A

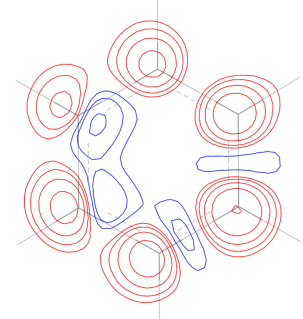

C

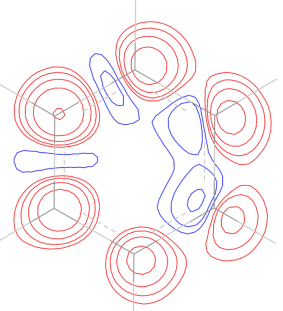

B

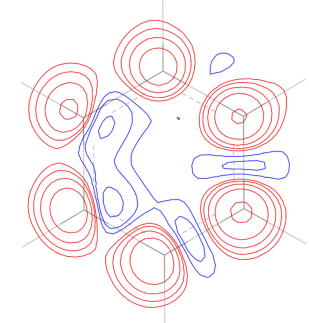

D

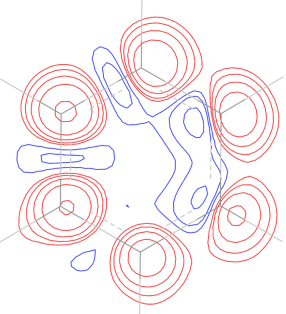

Figure 4. Two-dimensional plots of the differences between X-ray constrained and unconstrained Spin-Coupled electron densities. Plots above the aromatic ring (in a plane parallel to and $0.5 \AA$ above the one defined by atoms $C 1, C 2$ and C6): (A) $\rho_{X C S C / \lambda=0.20}-\rho_{S C / \lambda=0.00}$, (B) $\rho_{X C S C / \lambda=0.40}-\rho_{S C / \lambda=0.00}$. Plots below the aromatic ring (in a plane parallel to and $0.5 \AA$ below the one defined by atoms C1, C2 and C6): (C) $\rho_{X C S C / \lambda=0.20}-\rho_{S C / \lambda=0.00}$, (B) (D) $\rho_{X C S C / \lambda=0.40}-$ $\rho_{S C / \lambda=0.00}$. For each plot, the contours are drawn at $\pm 1 \cdot 10^{-3} \mathrm{e} / \mathrm{bohr}^{3}$ and at $\pm 2,4,8 \cdot 10^{n}$ e/bohr ${ }^{3}$ (with $n$ as an integer ranging from -3 to 0 ). Red and blue contours indicate positive and negative values, respectively.

To summarize, here we have proposed the first fully VB-like Xray constrained wavefunction method. The new technique allows the extraction of chemical information (e.g., weights of the resonance structures, spatial extensions of the electronic clouds around the atoms) from X-ray diffraction data without introducing preliminary information a priori or performing bond-analyses a posteriori. Our preliminary test calculations on benzene have shown that the new technique is indeed able to significantly capture the crystal field effects on the electronic structure, as testified by the observed differences in the obtained SpinCoupled orbitals, by the variation of the resonance structure weights and by the global changes in the electron density distribution. Furthermore, since the Spin-Coupled wavefunctions are exact spin-eigenfunctions also for open-shell systems, we envisage the further extension of the method to perform simultaneous refinements of X-ray and Polarized Neutron diffraction data, as alternative to the strategies already devised both in the framework of the multipole models of charge density $^{[15]}$ and in the context of the wavefunction-based techniques $^{\left[{ }^{[\mathrm{h}]}\right]}$ to obtain experimental spin densities. Finally, it is also worth noting that, if we exclude the very preliminary $\mathrm{XC}$ ELMO-VB strategy, the new method can be really considered as the first multi-determinant $\mathrm{X}$-ray constrained wavefunction method. For this reason, the new approach could be also exploited in the near future to extract two-electron density matrices from scattering experiments, but also to investigate more precisely if the XCW approach is able to capture electron correlation from $X$-ray diffraction data, a still open problem that so far, has been faced only in an effective way by mainly analyzing the effects of the wavefunction fitting on the electron density. ${ }^{[16]}$

\section{Acknowledgements}

The authors gratefully thank Mark Spackman and Hans-Beat Bürgi for providing the experimental X-ray diffraction data associated with the crystallographic structure of benzene. A.G. acknowledges the French Research Agency (ANR) for financial support through Grant No. ANR-17-CE29-0005.

Keywords: Quantum Crystallography - X-ray constrained wavefunction - X-ray diffraction • Valence Bond theory $\cdot$ SpinCoupled method

[1] a) R. F. Stewart, Acta Crystallogr., Sect. A 1976, 32, 565-574; b) N. K. Hansen, P. Coppens, Acta Crystallogr., Sect. A 1978, 34, 909-921.

[2] a) A. Genoni, L. Bučinský, N. Claiser, J. Contreras-García, B. Dittrich, P. M. Dominiak, E. Espinosa, C. Gatti, P. Giannozzi, J.-M. Gillet, D. Jayatilaka, P. Macchi, A. Ø. Madsen, L. J. Massa, C. F. Matta, K. M. Merz Jr., P. N. H. Nakashima, H. Ott, U. Ryde, K. Schwarz, M. Sierka, S. Grabowsky, Chem. Eur. J. 2018, 24, 10881-10905; b) S. Grabowsky, A. Genoni, H.-B. Bürgi, Chem. Sci. 2017, 8, 4159-4176; c) L. Massa, C. F. Matta, J. Comput. Chem. 2017, 39, 1021-1028; d) V. Tsirelson, J. Comput. Chem. 2017, 39, 1029-1037.

[3] a) D. Jayatilaka, Phys. Rev. Lett. 1998, 80, 798-801; b) D. Jayatilaka, D. J. Grimwood, Acta Crystallogr., Sect. A 2001, 57, 76-86; c) D. J. Grimwood, D. Jayatilaka, D. Acta Crystallogr., Sect. A 2001, 57, 87-100; d) I. Bytheway, D. Grimwood, D. Jayatilaka, Acta Crystallogr., Sect. A 2002, 58, 232-243; e) I. Bytheway, D. J. Grimwood, B. N. Figgis, G. S. Chandler, D. Jayatilaka, Acta Crystallogr., Sect. A 2002, 58, 244-251; f) D. J. Grimwood, I. Bytheway, D. Jayatilaka, J. Comput. Chem. 2003, 24, 470-483.

[4] a) W. L. Clinton, L. J. Massa, Phys. Rev. Lett. 1972, 29, 1363-1366; b) L. Massa, M. Goldberg, C. Frishberg, R. F. Boehme, S. J. La Placa, Phys. Rev. Lett. 1985, 55, 622-625; c) Y. V. Aleksandrov, V. G. Tsirelson, I. M. Reznik. R. P. Ozerov, Phys. Status Solidi B 1989, 155, 201-207; d) D. E. Hibbs, S. T. Howard, J. P. Huke, M. P. Waller, Phys. Chem. Chem. Phys. 2005, 7, 1772-1778; e) H. Schmider, V. H. Smith Jr., W. Weyrich, J. Chem. Phys. 1992, 96, 8986-8994; f) J.-M. Gillet, P. J. Becker, J. Phys. Chem. Sol. 2004, 65, 2017-2023; g) J.-M. Gillet, Acta Crystallogr., Sect. A 2007, 63, 234-238; h) S. Guedidda, Z. Yan, I. Kibalin, A. B. Voufack, N. Claiser, M. Souhassou, C. Lecomte, B. Gillon, J.-M. Gillet, J. Chem. Phys. 2018, 148, 164106.

[5] a) M. Hudák, D. Jayatilaka, L. Perašínova, S. Biskupic, J. Kozísek, L. Bučinský, Acta Crystallogr. Sect. A 2010, 66, 78-92; b) L. Bučinský, D. Jayatilaka, S. Grabowsky, J. Phys. Chem. A 2016, 120, 6650-6669.

[6] a) D. Jayatilaka, D. J. Grimwood, Acta Crystallogr., Sect. A 2004, 60, 111 - 119; b) S. Grabowsky, D. Jayatilaka, S. Mebs, P. Luger, Chem. Eur. J. 2010, 16, 12818-12821.

[7] a) M. Fugel, F. Fleemiss, L. A. Malaspina, R. Pal, P. R. Spackman, D. Jayatilaka, S. Grabowsky, Aust. J. Chem. 2018, 71, 227-237; b) M. Fugel, J. Beckmann, D. Jayatilaka, G. V. Gibbs, S. Grabowsky, Chem. Eur. J. 2018, 24, 6248-6261.

[8] a) A. Genoni, J. Phys. Chem. Lett. 2013, 4, 1093-1099; b) A. Genoni, J. Chem. Theory Comput. 2013, 9, 3004-3019; c) L. H. R. Dos Santos, A. Genoni, P. Macchi, Acta Crystallogr., Sect. A 2014, 70, 532-551; d) A. Genoni, B. Meyer, Adv. Quantum Chem. 2016, 73, 333-362.

[9] a) A. Genoni, Acta Crystallogr., Sect. A 2017, 73, 312-316; b) N. Casati, A. Genoni, B. Meyer, A. Krawczuk, P. Macchi, Acta Crystallogr., Sect. B 2017, 73, 584-597. 
[10] a) H. Stoll, G. Wagenblast, H. Preuss, Theor. Chim. Acta 1980, 57 169-178; b) A. Fornili, M. Sironi, M. Raimondi, J. Mol. Struct. (THEOCHEM) 2003, 632, 157-172; c) A. Genoni, M. Ghitti, S. Pieraccini, M. Sironi, Chem. Phys. Lett. 2005, 415, 256-260; d) M. Sironi, A. Genoni, M. Civera, S. Pieraccini, M. Ghitti, Theor. Chem. Acc. 2007, 117, 685-698; e) M. Sironi, M. Ghitti, A. Genoni, G. Saladino, S. Pieraccini, J. Mol. Struct. (THEOCHEM) 2009, 898, 8-16; f) B. Meyer B. Guillot, M. F. Ruiz-Lopez, A. Genoni, J. Chem. Theory. Comput. 2016, 12, 1052-1067; g) B. Meyer, B. Guillot, M. F. Ruiz-Lopez, C Jelsch, A. Genoni, J. Chem. Theory. Comput. 2016, 12, 1068-1081.

[11] a) J. Gerratt, W. N. Lipscomb, Proc. Natl. Acad. Sci. USA, 1968, 59, 332-335; b) J. Gerratt, Adv. Atom Mol. Phys. 1971, 7, 141-221; c) D. L. Cooper, J. Gerrat, M. Raimondi, M. Nature 1986, 323, 699-701; d) D. L. Cooper, J. Gerratt, M. Raimondi in Advances in the Theory of Benzenoid Hydrocarbons. Topics in Current Chemistry, Vol 153 (Eds.: I. Gutman, S. J. Cyvin), Springer, Berlin \& Heidelberg, 1990, pp. 41-55; e) D. L. Cooper, J. Gerratt, M. Raimondi, M. Sironi, T. Thorsteinsson, Theor. Chim. Acta 1993, 85, 261-270.

[12] P. C. Hiberty, S. Shaik, J. Comput. Chem. 2007, 28, 137-151.

[13] B. H. Chirgwin, C. A. Coulson, Proc. R. Soc. London A 1950, 201, 196209.

[14] H. B. Bürgi, S. C. Capelli, A. E. Goeta, J. A. C. Howard, M. A. Spackman, D. S. Yufit, Chem. Eur. J. 2002, 8, 3512-3521.

[15] a) M. Deutsch, N. Claiser, S. Pillet, Y. Chumakov, P. Becker, J.-M. Gillet, B. Gillon, C. Lecomte, M. Souhassou, Acta Crystallogr., Sect. A 2012, 68, 675-686; b) M. Deutsch, B. Gillon, N. Claiser, J.-M. Gillet, C. Lecomte, M. Souhassou, IUCrJ 2014, 1, 194-199; c) A. B. Voufack, N. Claiser, C. Lecomte, S. Pillet, Y. Pontillon, B. Gillon, Z. Yan, J.-M. Gillet, M. Marazzi, A. Genoni, M. Souhassou, Acta Crystallogr., Sect. B 2017, 73, 544-549.

[16] a) A. Genoni, L. H. R. Dos Santos, B. Meyer, P. Macchi, IUCrJ 2017, 4, 136-146; b) S. Grabowsky, Acta Crystallogr., Sect. A 2017, 73, C568. 


\section{Entry for the Table of Contents}

Layout 1:

\section{COMMUNICATION}

From X-ray diffraction to resonance structures weights. A new quantum crystallographic method that enables to take into account the X-ray diffraction data in fully Valence Bond calculations is proposed. Exploiting the new technique, insights into the electron distributions around each atomic center and, particularly, into the weights of resonance structures can be directly obtained from usual X-ray diffraction measurements.

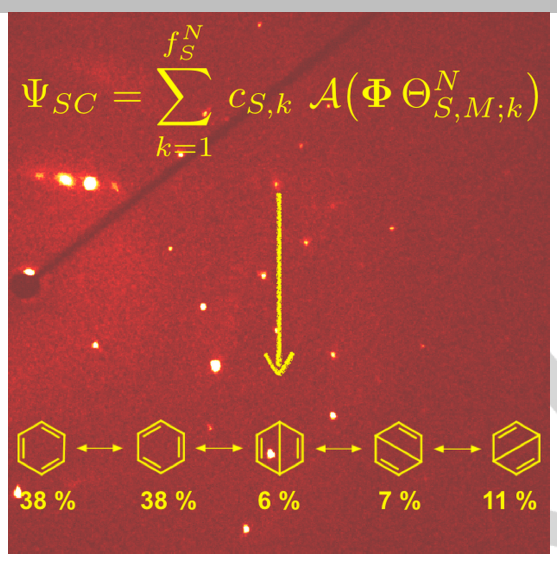

A. Genoni*, D. Franchini, S. Pieraccini, M. Sironi

Page No. - Page No.

X-ray constrained Spin-Coupled wavefunction: a tool to extract

chemical information from X-ray diffraction data 